\title{
Enhancing and Controlling Plasmons in Janus MoSSe-Graphene Based van der Waals Heterostructures
}

Cavalcante, L. S. R.; Gjerding, M. N.; Chaves, Andrey; Thygesen, K. S.

Published in:

Journal of Physical Chemistry C

Link to article, DOI:

10.1021/acs.jpcc.9b04000

Publication date:

2019

Document Version

Early version, also known as pre-print

Link back to DTU Orbit

Citation (APA):

Cavalcante, L. S. R., Gjerding, M. N., Chaves, A., \& Thygesen, K. S. (2019). Enhancing and Controlling Plasmons in Janus MoSSe-Graphene Based van der Waals Heterostructures. Journal of Physical Chemistry $C$, 123(26), 16373-16379. https://doi.org/10.1021/acs.jpcc.9b04000

\section{General rights}

Copyright and moral rights for the publications made accessible in the public portal are retained by the authors and/or other copyright owners and it is a condition of accessing publications that users recognise and abide by the legal requirements associated with these rights.

- Users may download and print one copy of any publication from the public portal for the purpose of private study or research.

- You may not further distribute the material or use it for any profit-making activity or commercial gain

- You may freely distribute the URL identifying the publication in the public portal 


\section{Enhancing and controlling plasmons in Janus}

\section{MoSSe-graphene based van der Waals}

\section{heterostructures}

L. S. R. Cavalcante, ${ }^{* \dagger}$ M. N. Gjerding, ${ }^{\ddagger}$ Andrey Chaves, ${ }^{\dagger}$ and K. S. Thygesen ${ }^{*, \ddagger}$

$\dagger$ Departamento de Física, Universidade Federal do Ceará, Caixa Postal 6030, Campus do Pici, 60455-900 Fortaleza, Ceará, Brazil

$\ddagger C A M D$, Department of Physics, Technical University of Denmark, 2800 Kgs. Lyngby, Denmark

E-mail: lucaskvalcante@fisica.ufc.br; thygesen@fysik.dtu.dk 


\begin{abstract}
We explore the use of MoSSe Janus layers, which possess an intrinsic electric dipole caused by their out-of-plane structural asymmetry, to selectively dope graphene embedded inside a heterostructure without the need of external sources (such as electrostatic gates or chemical functionalization), in order to engineer graphene plasmons. Using the quantum-electrostatic heterostructure method, we demonstrate that through the control of the plasmon energy via the doping level and the hybridization of plasmons in different layers, we can reach graphene plasmon energies up to $0.5 \mathrm{eV}$ or selectively quench certain (symmetric) modes by Landau damping. The possibility of using other Janus transition metal dichalcogenides that could improve this effect is also investigated.
\end{abstract}

\title{
Introduction
}

Van der Waals heterostructures ${ }^{1}$ (vdWHs) open the possibility to combine various properties of layered materials to achieve new applications. ${ }^{2-9}$ An interesting combination is the one between graphene, which presents Dirac cones in the low energy range of its band structure, ${ }^{10}$ and transition metal dichalcogenides (TMDs), which exhibit a direct band gap in monolayer form. ${ }^{11}$

Graphene is a carbon based two-dimensional (2D) material composed by a single layer of atoms arranged in a hexagonal lattice. On the other hand, TMDs have $\mathrm{MX}_{2}$ form, with a metal (M) layer sandwiched by two layers of a chalcogen (X). ${ }^{12}$ Recently, a new prototype of TMDs called Janus structures ${ }^{13}$ have attracted considerable attention. ${ }^{14-17}$ In this structure, the TMD reflection symmetry in the out-of-plane $(z$-)direction is broken by changing one layer of chalcogens $\mathrm{X}$ in $\mathrm{MX}_{2}$ by a layer of another chalcogen $\mathrm{Y}$, thus leading to MXY. This results in different electronegativity for each side, which induces an out-of-plane dipole that is intrinsic to the system. This feature, in combination with the stacking of semimetalic electrodes (such as graphene), creates new possibilities for applications as p-n junctions that 
are doped without the need of any external interaction. ${ }^{18}$ Knowing the energy difference across the Janus structure, it is possible to easily control the graphene doping by changing the number of Janus TMDs layers stacked in the system.

In this work, we investigate the effect of stacking MoSSe layers (our Janus TMDs of choice) intercallated with graphene layers, on their plasmonic states. ${ }^{19-24}$ The choice of MoSSe as a Janus material is motivated by its recent experimental synthesis, starting from $\mathrm{MoS}_{2}{ }^{13}$ or $\mathrm{MoSe}_{2} \cdot{ }^{25}$ Several properties of this monolayer, including optical and magnetic properties, have already been studied in the literature. ${ }^{26,27}$ Our results show that, by means of structure engineering, plasmon energies can be controlled, reaching values up to $0.5 \mathrm{eV}$. Furthermore, we demonstrate that it is possible to selectively quench certain branches of the plasmon dispersion. Finally, we investigate other Janus TMDs candidates that could improve the effects discussed here, by adjusting the relation between the dipole shift and band gap.

\section{Computational Methods}

The quantum-electrostatic heterostructure $(\mathrm{QEH})^{28}$ model is employed to calculate the plasmonic properties in different graphene-MoSSe heterostructures. Within this model, the dielectric response of the component layers is calculated independently with density functional theory (DFT) and the total response of the heterostructure is then built by coupling the monolayers together by the Coulomb interaction. Using this model, one can efficiently calculate the dielectric properties of vdWHs with ab initio precision.

However, resolving the low energy linearly dispersive bands of graphene introduces a practical problem for DFT calculations, namely, that a high density of $k$ points is necessary to properly describe its physical properties, and this greatly increases the computational costs. To overcome this problem, we used an analytical solution for the doped graphene density response function. We start by calculating the non-interacting $2 \mathrm{D}$ polarizability ${ }^{29}$ for 
a finite doping, and then we express it within the relaxation-time (RT) approximation to take into account possible extrinsic processes. ${ }^{30}$ Finally, using the Random Phase Approximation (RPA), we derive the full interacting density response function. We combine the analytical solution of the density response function with the DFT results for the induced density, that is well-defined even for a low density of $k$ points, thus creating a hybrid graphene dielectric building block for QEH calculations.

\section{Results and Discussion}

Let us first study a system composed by MoSSe layers encapsulated by graphene, as illustrated in the inset in Fig. 1. As discussed in the Introduction, the intrinsic dipole created by the asymmetry in electronegativity between the $\mathrm{S}$ and the Se atoms of the MoSSe layer induces an electric field that creates $\mathrm{n}$ - and p-type doping on the top and bottom graphene layers, respectively. This system resembles a capacitor and, in what follows, we demonstrate that the dependence of its doping level on the number of MoSSe layers can be described by a simple model that requires only a few material-specific parameters.

The total electric field, $\mathbf{E}=\mathbf{E}_{\text {MoSSe }}+\mathbf{E}_{\text {depol }}$, is defined as the sum of the electric field created by the intrinsic dipole of the MoSSe layers

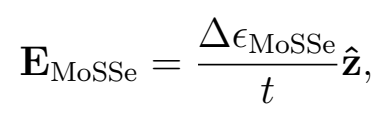

in which $\Delta \epsilon_{\text {MoSse }}$ is the energy difference generated by the built-in dipole, also known as the dipole shift, $t$ is the thickness of the MoSSe layer, and the depolarization field generated by the induced density on the graphene layers,

$$
\mathbf{E}_{\mathrm{depol}}=-\frac{\sigma}{2 \epsilon \epsilon_{0}} \hat{\mathbf{z}}
$$

where $\sigma$ represents the graphene induced density, given by $\sigma=\epsilon_{\mathrm{F}}^{2} / \pi \hbar^{2} v_{\mathrm{F}}^{2}$. The effective 
dielectric constant in the $z$ direction in MoSSe is assumed to be $\epsilon=1+4 \pi \alpha_{3 \mathrm{D}}$, where $\alpha_{3 \mathrm{D}}$ is the average long-wavelength polarizability of bulk MoSSe, which is obtained from its monolayer static polarizability as $\alpha_{3 \mathrm{D}}=\alpha_{2 \mathrm{D}} / t$.

Hence, modeling the doping in the system by assuming a Fermi energy

$$
2 \epsilon_{\mathrm{F}}=E d
$$

where $d$ is the distance between the plates of an analogous capacitor, which is simply given by the product of the number of MoSSe layers $\mathrm{N}$ and the thickness $t$, we finally obtain

$$
\epsilon_{\mathrm{F}}=\frac{-1+\sqrt{1+a N^{2} \Delta_{\mathrm{MoSSe}}}}{a N}
$$

with

$$
a=\frac{t}{2 \epsilon \epsilon_{0} \pi \hbar^{2} v_{\mathrm{F}}^{2}}
$$

In Fig. 1, we compare the results from such capacitor model with those from DFT calculations ${ }^{18}$ for the dependence of the graphene doping on the number of MoSSe layers, considering the system shown in the inset, assuming $t=6.4 \AA, \Delta_{\text {MoSSe }}=0.51 \mathrm{eV}$ and $\epsilon=1.76$. Good agreement between the results is observed even for a high number of layers, where the doping level reaches an upper bound due to the increasing strength of the depolarization field, which ends up cancelling out the dipole shift of the MoSSe layers. Such good agreement between the results from ab initio and the capacitor model gives us confidence that the latter would also apply in more complex heterostrutures, where DFT calculations would be impractical.

In order to demonstrate the effectiveness of the graphene doping achieved by stacking it with MoSSe, we have calculated the loss function $\mathcal{L}=-\operatorname{Im}\left\{\epsilon_{\mathrm{RPA}, \mathrm{RT}}^{-1}(q, w)\right\}$, for a monolayer of MoSSe encapsulated by doped graphene. Results are presented in Fig. 2(a), which exhibits two plasmon branches: a symmetric optical plasmon, with higher frequency, and 


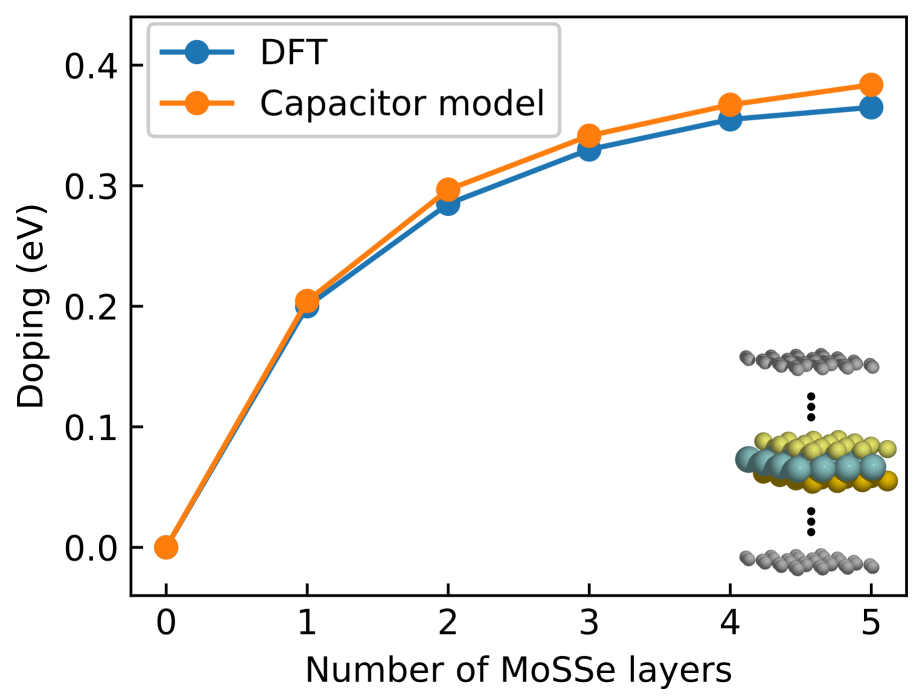

Figure 1: Graphene doping level as a function of the number of MoSSe layers, for the structure illustrated in the inset, as calculated using DFT (blue) and the capacitor model (orange), with $t=6.4 \AA, \Delta_{\mathrm{MoSSe}}=0.51 \mathrm{eV}$ and $\epsilon=1.76$.

an antisymmetric acoustic plasmon, with lower frequency. The (anti)symmetry across the system is verified by its induced density, in Fig. 2(b) for $q=0.01 \AA$.

As a consequence of the previously mentioned compensating depolarization field, increasing the number of MoSSe layers beyond $N \approx 6$ does not lead to a significant increase in the doping level of the graphene layers, which ultimately limits the achievable plasmon energies. In order to circumvent this problem, one could take advantage of the fact that the direction of the intrinsic dipole is defined by the relative ordering of sulfur and selenium atoms in the MoSSe structure, and thus stack more layers with opposite ordering surrounding a middle graphene layer, as represented in the inset of Fig. 3. This increases the maximum achievable doping level of the middle graphene layer beyond the threshold of the bilayer graphene-MoSSe heterostructure, as one can see by the higher doping level in the middle graphene layer in Fig. 3.

In order to calculate the graphene doping as a function of the number of MoSSe layers for this new type of structure, we again use the capacitor model considering one of the outer graphene layers together with the middle graphene layer. The middle graphene layer is expected to support twice the charge density of each of the outer graphene layers, due 

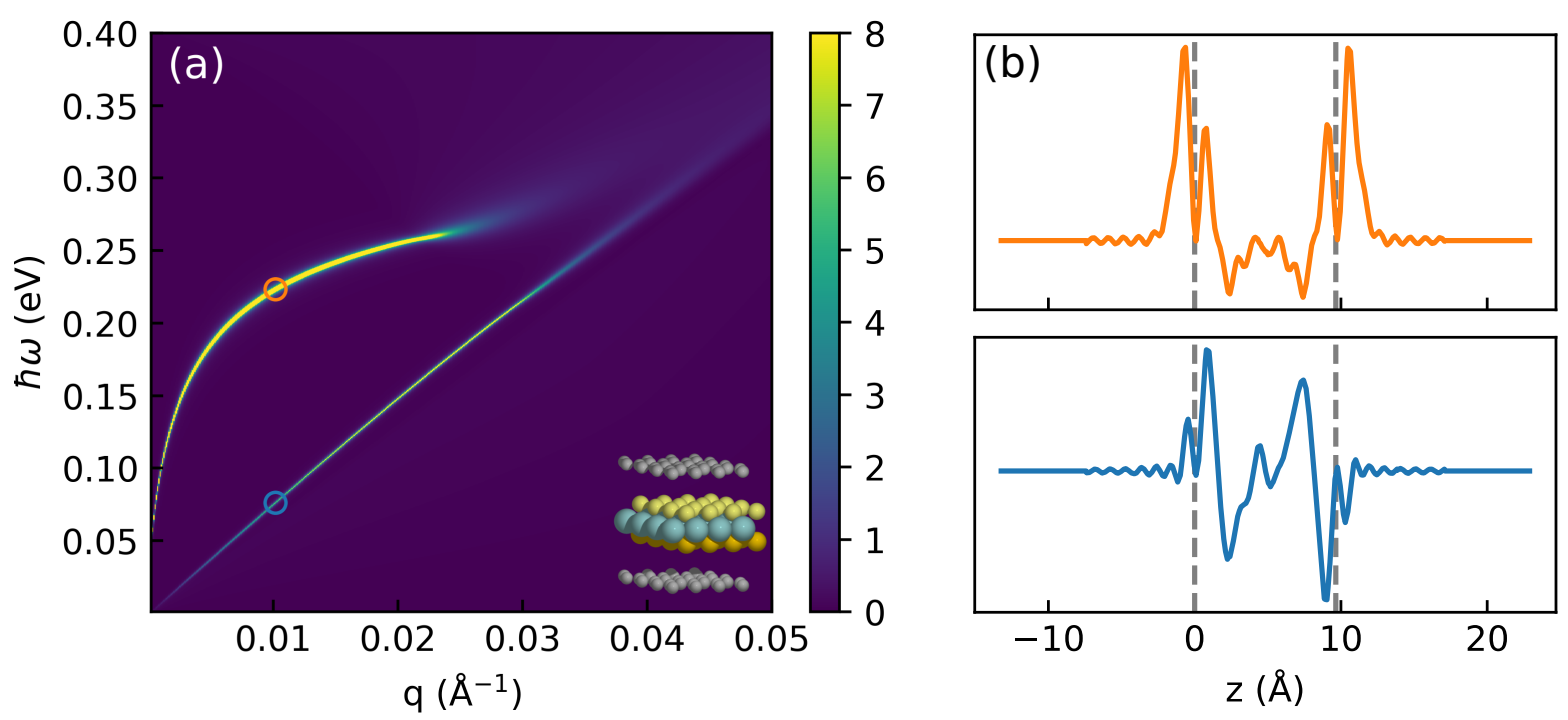

Figure 2: (a) Loss function for monolayer of MoSSe encapsulated by graphene, as illustrated in the inset, with $\epsilon_{F}=0.2 \mathrm{eV}$. Induced density presents one symmetric and one antisymmetric mode, as shown in (b) for the higher (top, orange) and lower (bottom, blue) energy branches at $q=0.01 \AA^{-1}$. The vertical dashed grey lines represent the graphene layers positions.

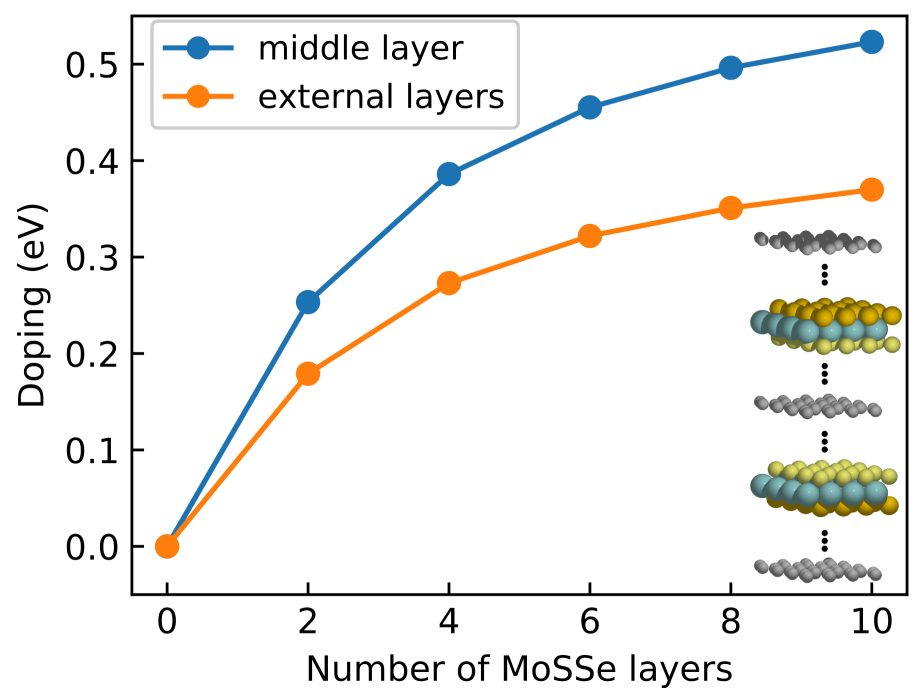

Figure 3: Doping level in the middle and external graphene layers of the structure illustrated in the inset, as a function of the number of MoSSe layers, as calculated using the capacitor model. 
to charge conservation. Hence, the energy difference between the outer and inner graphene layers, instead of $2 \epsilon_{F}$ in Eq. $(3)$, becomes $(1+\sqrt{2}) \epsilon_{F}$. Consequently, we obtain

$$
\epsilon_{\mathrm{F}}=\frac{-1-\sqrt{2}+\sqrt{3+2 \sqrt{2}+4 a N^{2} \Delta_{\mathrm{MoSSe}}}}{2 a N} .
$$

Considering only two layers of MoSSe with opposing dipole orientations, the calculated loss spectrum and the induced densities corresponding to the plasmon excitations are shown Fig. 4. There are now three doped graphene layers, which reflect in three branches of the loss function, of which are two symmetric and one antisymmetric for $q=0.01 \AA$. Besides the addition of a new acoustic mode in the spectrum, there are no major differences in the plasmon energies in comparison to the first case presented in Fig. 2. The reason for this similarity can be understood by noticing that the actual doping level in the inner graphene layer is about $250 \mathrm{meV}$, which is not significantly larger than the bilayer graphene-MoSSe heterostructure, which exhibits a doping level of $\approx 200 \mathrm{meV}$.
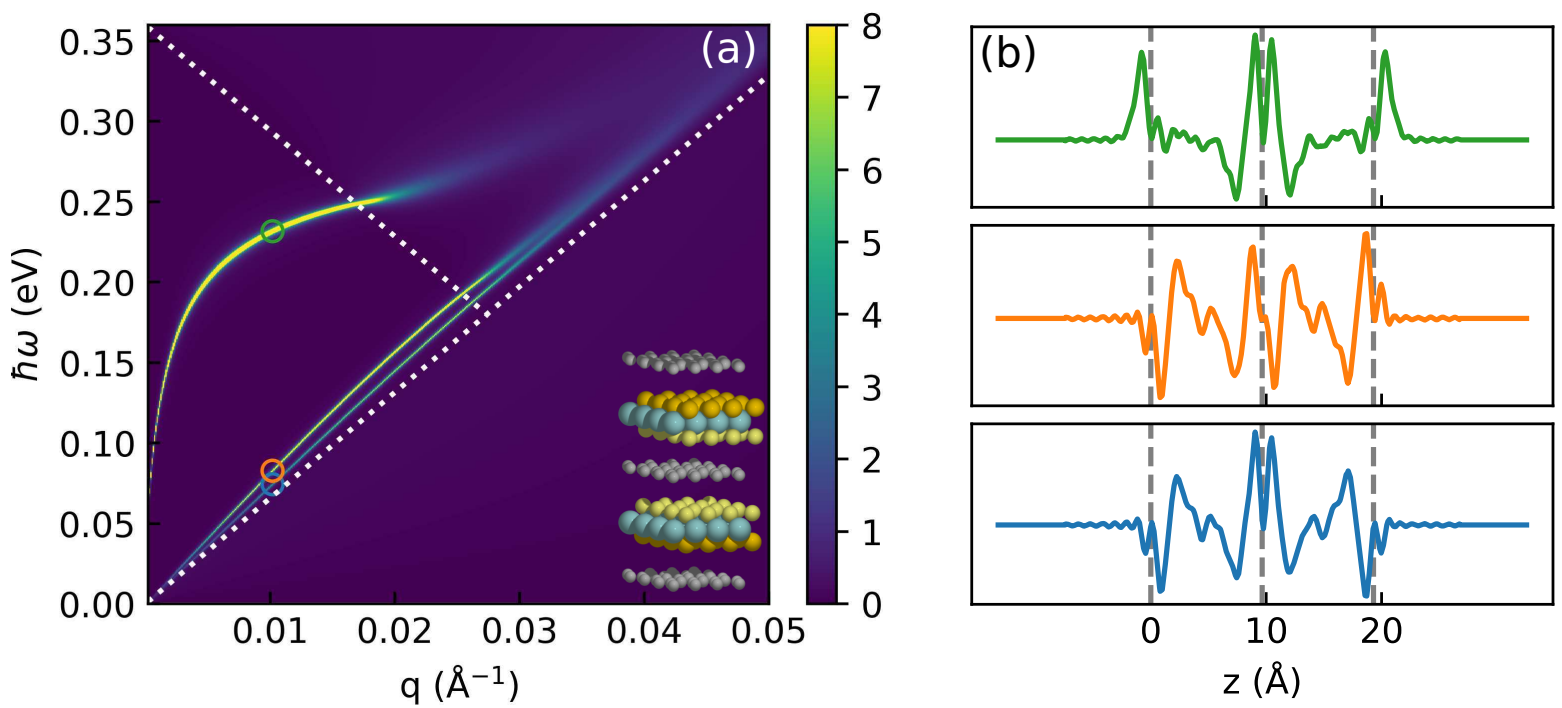

Figure 4: (a) Loss function for the heterostructure illustrated in the inset, composed by two external graphene layers $\left(\epsilon_{\mathrm{F}}=0.18 \mathrm{eV}\right)$, one middle graphene layer $\left(\epsilon_{\mathrm{F}}=0.25 \mathrm{eV}\right)$ and two layers of MoSSe with opposite orientation. White dashed lines delimit the region of Landau damping of the plasmon modes. (b) The induced densities for $q=0.01 \AA^{-1}$ now present three modes: two symmetric and one antisymmetric. The vertical dashed grey lines represent the graphene layers positions. 
We now compare the more extreme cases for both bilayer graphene heterostructure with 5 layers of MoSSe and trilayer graphene heterostructures with five layers of MoSSe sandwiched between each of the graphene layer. The results are shown in Fig. 5. In the latter, the new symmetric plasmon branch that appears achieves much higher frequencies, namely up to 0.5 $\mathrm{eV}$, without undergoing damping, which is simply a consequence of the increased doping of the middle layer. As indicated in the figure, the highest energy plasmon mode actually extends into the Landau damping regime of the outer graphene layers without suffering losses, which can be explained by the fact that the plasmon is localized in the middle graphene layer and thus cannot excite electron-hole pairs in the outer ones. Furthermore, the upper limit of the achieved plasmon energy is determined mainly by the point where the plasmon branch enters the Landau damping regime that is directly related to the Fermi energy of the layer where it is localized. Therefore, our results show that in such a MoSSe/graphene van der Waals heterostructure, it is possible to obtain plasmon energies that are among the highest ever reported, ${ }^{20,31-33}$ without the need of external doping sources, such as electrostatic gates or chemical functionalization.
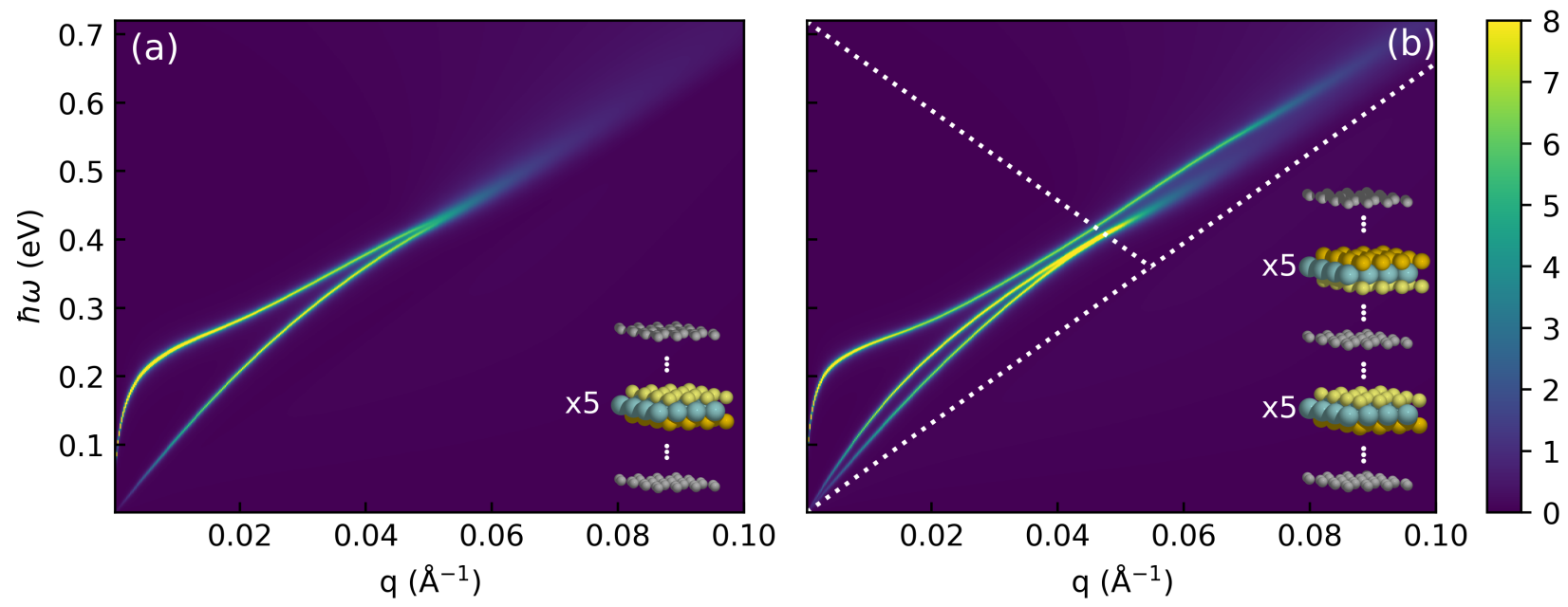

Figure 5: Comparison between loss functions of heterostructures composed by (a) five layers of MoSSe encapsulated by two graphene layers $\left(\epsilon_{\mathrm{F}}=0.36 \mathrm{eV}\right)$ and (b) ten layers of MoSSe, divided into two stacks of MoSSe with opposite orientations, intercalated and encapsulated by three layers of graphene $\left(\epsilon_{\mathrm{F} \text {,external }}=0.36 \mathrm{eV}\right.$ and $\left.\epsilon_{\mathrm{F} \text {,middle }}=0.50 \mathrm{eV}\right)$. White dashed lines delimit the Landau damping region of the plasmon modes of the outer graphene layers. 
A second type of heterostructure, also with three layers of graphene, but assuming all MoSSe layers with the same dipole orientation (see the inset in Fig. 6(a)) is now investigated. In this case, only the outer graphene layers become doped, while the middle graphene layer stays undoped. While the structure is, in principle, similar to the previously discussed bilayer graphene-MoSSe heterostructure, the addition of the middle graphene layer adds significant losses in the form of Landau damping for all frequencies and momenta. As a consequence, the optical mode is quenched in contrast to the acoustic mode, which survives. The origin of the difference in losses of the two modes is found in the associated electric fields of the modes: the optical mode is associated with a large in-plane electric field on the middle undoped graphene layer which excites many electron hole pairs, whereas the acoustic mode exhibits an out-of-plane electric field that cannot excite electron-hole pairs and is therefore not susceptible to the losses introduced by the middle graphene layer. The quenching of the optical mode can be further increased, as verified in Fig. 6(b) by adding a second undoped graphene layer to the middle of the heterostructure. These results show that it is possible to selectively engineer the quenching of optical modes in graphene heterostructures, leaving the acoustic modes as the only propagating modes in the structure.
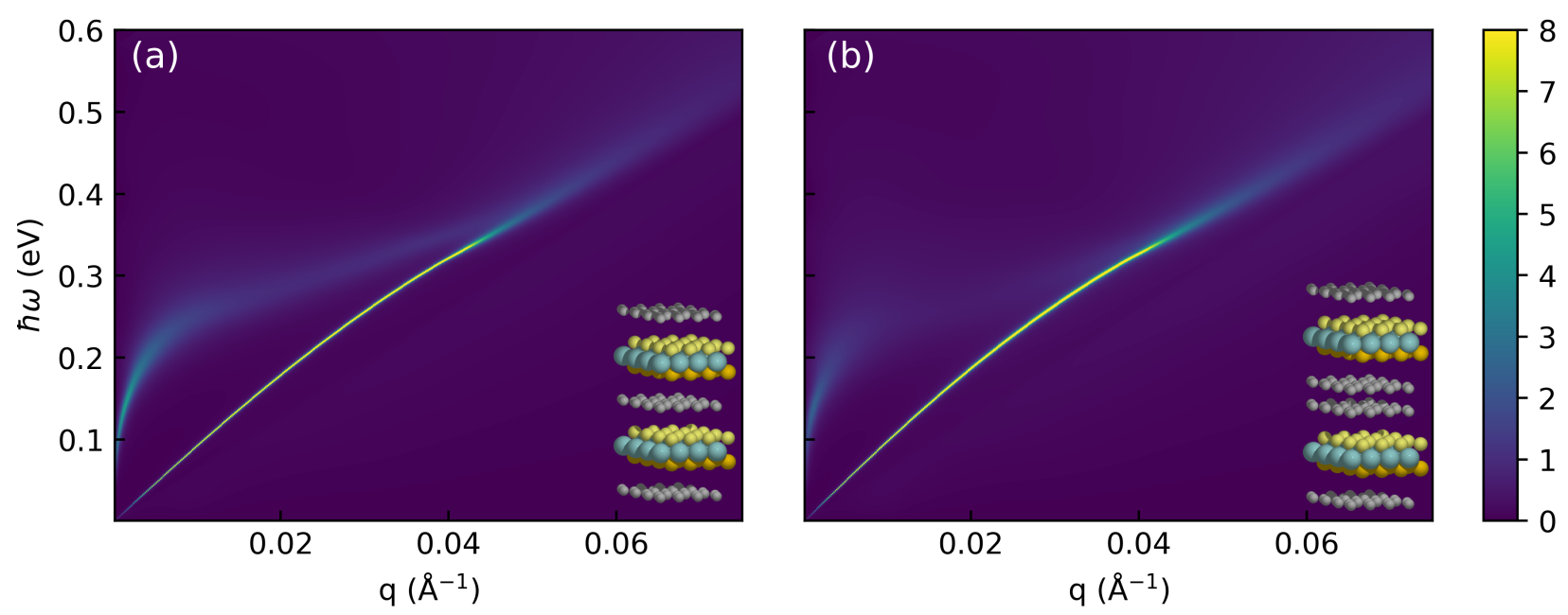

Figure 6: Loss function for two MoSSe layers encapsulated by graphene $\left(\epsilon_{\mathrm{F}}=0.29 \mathrm{eV}\right)$ where we add one (a) and two (b) layer(s) of graphene in the middle of the heterostructure.

This work opens new possibilities of engineering plasmons in $\mathrm{p}-\mathrm{n}$ junctions that are 
created from the stacking of Janus TMDs using graphene leads. Besides MoSSe, we believe that it is possible to use other Janus TMDs that have greater dipole shifts to create plasmons with even higher energies than those presented here, without any type of external doping. The relation between doping and the optical plasmon energy can be expanded for small momenta to yield (see Supporting Information)

$$
\hbar \omega_{\mathrm{opt}} \approx e \sqrt{\frac{N \Delta_{\mathrm{Janus}}}{2 \pi \epsilon_{0}}} \sqrt{q},
$$

where the energy of the optical mode is derived in the non-retarded limit $(q \gg \sqrt{\epsilon} \omega / c)$ with the Fermi energy defined as in Eq. 4 for the structure presented in the inset of Fig. 1. One verifies that the optical plasmon energy directly depends on the dipole shift $\Delta_{\text {Janus }}$ of the Janus TMD that compose the structure, but is not affected by the screening of the dielectric environment, due to compensating effects between the additional screening and increased charge density in graphene, which also scales with $\epsilon$.

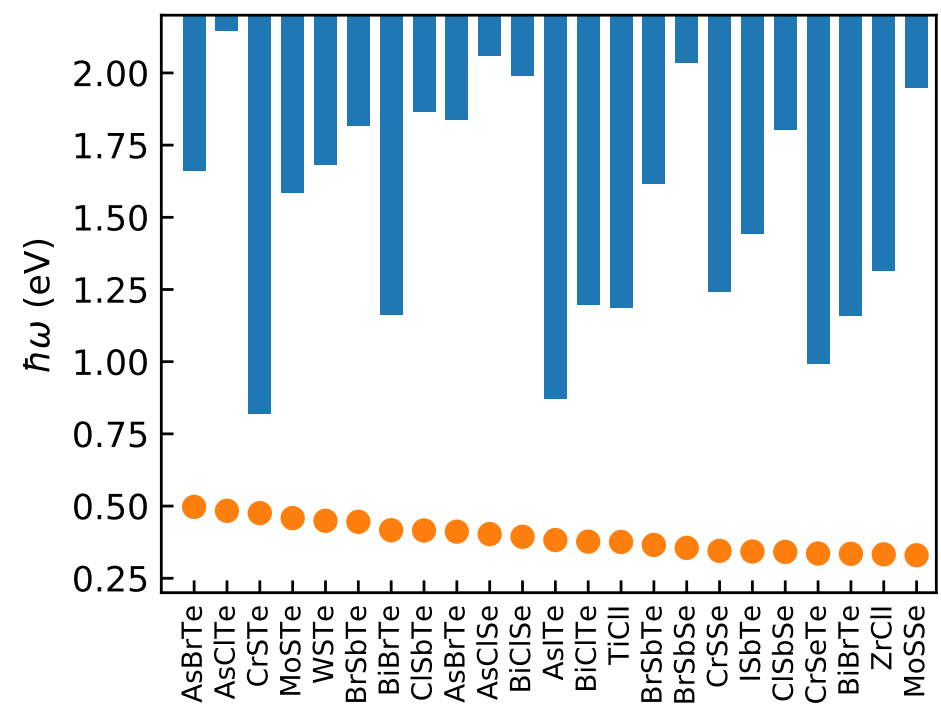

Figure 7: Optical plasmon energies (orange circles) for different Janus TMDs composing the structure sketched on the inset of Fig. 5 (a), for $q=0.001 \AA$. Electron-hole pair continuum for each material is shown by blue bars.

Optical plasmon energies, as calculated with Eq. (7) for a heterostructure with five TMD layers encapsulated by graphene (see sketch in Fig. 5 (a)) at $q=0.001 \AA$ are shown in Fig. 
7, for several stable Janus materials. All these Janus materials are highly thermodynamically stable, and are dynamically stable with respect to rearrangements of the atoms that preserve the layered character of the material. We stress this because the dynamic stability analysis will not take into account the ability of the materials 'roll up' due to the different sizes of Xand Y- atoms. ${ }^{34}$ The Janus materials have higher $\Delta_{\text {Janus }}$ as compared to MoSSe, ${ }^{35}$ which could improve the p-n junction properties by enhancing the plasmon energies. Equation (7) is expected to break down when the plasmon energies approach the band gap, which would thus screen and reduce the plasmon energy. In Fig. (7), the band gap and the onset of interband transitions are indicated by blue bars, which shows that, for some materials, the band gap is considerably large as compared to the unscreened plasma frequency. Based on this, we propose Arsenic Tellurium Chloride (AsTeCl) as the most promising Janus few layer system for plasmonics, with a high band gap energy.

\section{Conclusions}

In summary, we have investigated the effect of the intrinsic electric dipole across a Janus TMD layer, on the doping level and plasmonic frequencies of graphene layers within a Janus TMD/graphene van der Waals heterostructure. A strong variation of the doping level in the graphene layers is observed as the number of TMD layers increase, especially for a graphene layer placed in between two Janus TMD stacks with opposite orientation. On the other hand, by aligning the orientation of the two Janus TMD stacks encapsulating a few layer graphene sheet, the graphene optical plasmonic modes become quenched, whereas acousting plasmon states stay visible up to very high energies. These results suggest that stacking Janus TMD layers intercalated with graphene is an efficient way to select graphene plasmon modes and provide strong doping without the need of external sources, such as electrostatic gates and chemical adsorption. Although results are obtained here specifically for the case of MoSSe, a systematic study of several other potentially synthesizable Janus structures indicate that 
other Janus monolayers, with larger band gaps and larger out-of-plane dipole moments than MoSSe, might be even more efficient for doping and controlling graphene plasmons.

\section{Acknowledgement}

This study was partially supported by the Coordenação de Aperfeiçoamento de Pessoal de Nível Superior - Brasil (CAPES) - Finance Code 001. K.S.T. acknowledges funding from the European Research Council (ERC) under the European Union's Horizon 2020 research and innovation program (Grant Agreement No. 773122, LIMA).

\section{References}

(1) Geim, A. K.; Grigorieva, I. V. Van der Waals heterostructures. Nature 2013, 499, 419-425.

(2) Raja, A.; Chaves, A.; Yu, J.; Arefe, G.; Hill, H. M.; Rigosi, A. F.; Berkelbach, T. C.; Nagler, P.; Schüller, C.; Korn, T. et al. Coulomb engineering of the bandgap and excitons in two-dimensional materials. Nature Communications 2017, 8, 15251.

(3) Basov, D. N.; Fogler, M. M.; García de Abajo, F. J. Polaritons in van der Waals materials. Science (New York, N.Y.) 2016, 354, aag1992.

(4) Wilson, N. P.; Yu, H.; Rivera, P.; Yao, W.; Xu, X.; Seyler, K. L. Interlayer valley excitons in heterobilayers of transition metal dichalcogenides. Nature Nanotechnology 2018, 13, 1004-1015.

(5) Novoselov, K. S.; Mishchenko, A.; Carvalho, A.; Castro Neto, A. H. 2D materials and van der Waals heterostructures. Science 2016, 353, aac9439.

(6) Liu, Y.; Weiss, N. O.; Duan, X.; Cheng, H. C.; Huang, Y.; Duan, X. Van der Waals heterostructures and devices. Nature Reviews Materials 2016, 1, 16042. 
(7) Cavalcante, L. S. R.; Chaves, A.; Van Duppen, B.; Peeters, F. M.; Reichman, D. R. Electrostatics of electron-hole interactions in van der Waals heterostructures. Physical Review B 2018, 97, 125427.

(8) Viti, L.; Hu, J.; Coquillat, D.; Politano, A.; Consejo, C.; Knap, W.; Vitiello, M. S. Heterostructured hBN-BP-hBN Nanodetectors at Terahertz Frequencies. Advanced Materials 2016, 28, 7390-7396.

(9) Mattheakis, M.; Valagiannopoulos, C. A.; Kaxiras, E. Epsilon-near-zero behavior from plasmonic Dirac point: Theory and realization using two-dimensional materials. Physical Review B 2016, 94, 201404.

(10) Novoselov, K. S.; Geim, A. K.; Guinea, F.; Peres, N. M. R.; Castro Neto, A. H. The electronic properties of graphene. Reviews of Modern Physics 2009, 81, 109-162.

(11) Mak, K. F.; Lee, C.; Hone, J.; Shan, J.; Heinz, T. F. Atomically Thin MoS 2 : A New Direct-Gap Semiconductor. Physical Review Letters 2010, 105, 136805.

(12) Strano, M. S.; Kis, A.; Coleman, J. N.; Wang, Q. H.; Kalantar-Zadeh, K. Electronics and optoelectronics of two-dimensional transition metal dichalcogenides. Nature Nanotechnology 2012, \%, 699-712.

(13) Lu, A. Y.; Zhu, H.; Xiao, J.; Chuu, C. P.; Han, Y.; Chiu, M. H.; Cheng, C. C.; Yang, C. W.; Wei, K. H.; Yang, Y. et al. Janus monolayers of transition metal dichalcogenides. Nature Nanotechnology 2017, 12, 744-749.

(14) Riis-Jensen, A. C.; Pandey, M.; Thygesen, K. S. Efficient Charge Separation in 2D Janus van der Waals Structures with Built-in Electric Fields and Intrinsic p-n Doping. The Journal of Physical Chemistry C 2018, 122, 24520-24526.

(15) Er, D.; Ye, H.; Frey, N. C.; Kumar, H.; Lou, J.; Shenoy, V. B. Prediction of En- 
hanced Catalytic Activity for Hydrogen Evolution Reaction in Janus Transition Metal Dichalcogenides. Nano Letters 2018, 18, 3943-3949.

(16) Guo, S.-D. Phonon transport in Janus monolayer MoSSe: a first-principles study. Physical Chemistry Chemical Physics 2018, 20, 7236-7242.

(17) Hu, T.; Jia, F.; Zhao, G.; Wu, J.; Stroppa, A.; Ren, W. Intrinsic and anisotropic Rashba spin splitting in Janus transition-metal dichalcogenide monolayers. Physical Review B 2018, 97, 235404.

(18) Markussen, T.; Brandbyge, M.; Palsgaard, M.; Gunst, T.; Thygesen, K. S. Stacked Janus Device Concepts: Abrupt pn-Junctions and Cross-Plane Channels. Nano Letters 2018, 18, 7275-7281.

(19) Grigorenko, A. N.; Polini, M.; Novoselov, K. S. Graphene plasmonics. Nature Photonics 2012, 6, 749-758.

(20) Low, T.; Avouris, P. Graphene Plasmonics for Terahertz to Mid-Infrared Applications. ACS Nano 2014, 8, 1086-1101.

(21) Constant, T. J.; Hornett, S. M.; Chang, D. E.; Hendry, E. All-optical generation of surface plasmons in-graphene. Nature Physics 2016, 12, 124-127.

(22) Koppens, F. H. L.; Chang, D. E.; Garcia de Abajo, F. J. Graphene Plasmonics: A Platform for Strong Light-Matter Interactions. Nano Letters 2011, 11, 3370-3377.

(23) Politano, A.; Chiarello, G. Plasmon modes in graphene: status and prospect. Nanoscale 2014, 6, 10927-10940.

(24) Shirodkar, S. N.; Mattheakis, M.; Cazeaux, P.; Narang, P.; Soljačić, M.; Kaxiras, E. Quantum plasmons with optical-range frequencies in doped few-layer graphene. Physical Review B 2018, 97, 195435. 
(25) Zhang, J.; Jia, S.; Kholmanov, I.; Dong, L.; Er, D.; Chen, W.; Guo, H.; Jin, Z.; Shenoy, V. B.; Shi, L. et al. Janus Monolayer Transition-Metal Dichalcogenides. ACS Nano 2017, 11, 8192-8198.

(26) Li, F.; Wei, W.; Zhao, P.; Huang, B.; Dai, Y. Electronic and Optical Properties of Pristine and Vertical and Lateral Heterostructures of Janus MoSSe and WSSe. Journal of Physical Chemistry Letters 2017, 8, 5959-5965.

(27) Meng, M.; Li, T.; Li, S.; Liu, K. Ferromagnetism induced by point defect in Janus monolayer MoSSe regulated by strain engineering. Journal of Physics D: Applied Physics 2018, 51, 105004 .

(28) Andersen, K.; Latini, S.; Thygesen, K. S. Dielectric Genome of van der Waals Heterostructures. Nano Letters 2015, 15, 4616-4621.

(29) Wunsch, B.; Stauber, T.; Sols, F.; Guinea, F. Dynamical polarization of graphene at finite doping. New Journal of Physics 2006, 8, 318-318.

(30) Miyahara, T. Undulators, Wigglers and Their Applications; Holt, Rinehart and Winston, 2010; pp 349-368.

(31) Bezares, F. J.; Sanctis, A. D.; Saavedra, J. R. M.; Woessner, A.; Alonso-González, P.; Amenabar, I.; Chen, J.; Bointon, T. H.; Dai, S.; Fogler, M. M. et al. Intrinsic PlasmonâĂŞPhonon Interactions in Highly Doped Graphene: A Near-Field Imaging Study. Nano Letters 2017, 17, 5908-5913.

(32) Yan, H.; Low, T.; Zhu, W.; Wu, Y.; Freitag, M.; Li, X.; Guinea, F.; Avouris, P.; Xia, F. Damping pathways of mid-infrared plasmons in graphene nanostructures. Nature Photonics 2013, 7, 394-399.

(33) Freitag, M.; Low, T.; Zhu, W.; Yan, H.; Xia, F.; Avouris, P. Photocurrent in graphene harnessed by tunable intrinsic plasmons. Nature Communications 2013, 4, 1951. 
(34) Luo, Y. F.; Pang, Y.; Tang, M.; Song, Q.; Wang, M. Electronic properties of Janus MoSSe nanotubes. Computational Materials Science 2019, 156, 315-320.

(35) Haastrup, S.; Strange, M.; Pandey, M.; Deilmann, T.; Schmidt, P. S.; Hinsche, N. F.; Gjerding, M. N.; Torelli, D.; Larsen, P. M.; Riis-Jensen, A. C. et al. The Computational 2D Materials Database: high-throughput modeling and discovery of atomically thin crystals. 2D Materials 2018, 5, 042002. 